\title{
A wavelet-based approach to detect climate change on the coherent and turbulent component of the atmospheric circulation
}

\author{
Davide Faranda and Dimitri Defrance \\ LSCE-IPSL, CEA Saclay l'Orme des Merisiers, CNRS UMR 8212 CEA-CNRS-UVSQ, \\ Université Paris-Saclay, 91191 Gif-sur-Yvette, France
}

Correspondence to: Davide Faranda (davide.faranda@lsce.ipsl.fr)

Received: 28 January 2016 - Published in Earth Syst. Dynam. Discuss.: 17 February 2016

Revised: 1 June 2016 - Accepted: 2 June 2016 - Published: 20 June 2016

\begin{abstract}
The modifications of atmospheric circulation induced by anthropogenic effects are difficult to capture because wind fields feature a complex spectrum where the signal of large-scale coherent structures (planetary, baroclinic waves and other long-term oscillations) is mixed up with turbulence. Our purpose is to study the effects of climate changes on these two components separately by applying a wavelet analysis to the $700 \mathrm{hPa}$ wind fields obtained in climate simulations for different forcing scenarios. We study the coherent component of the signal via a correlation analysis to detect the persistence of large-scale or long-lasting structures, whereas we use the theory of autoregressive moving-average stochastic processes to measure the spectral complexity of the turbulent component. Under strong anthropogenic forcing, we detect a significant climate change signal. The analysis suggests that coherent structures will play a dominant role in future climate, whereas turbulent spectra will approach a classical Kolmogorov behaviour.
\end{abstract}

\section{Introduction}

Scale separation is an essential property for the study of natural systems: Lagrangian mechanics has been applied to the study of the solar system because planets appear so small that they can be considered to be material points with respect to the length of their orbits (Murray and Dermott, 1999). In a less obvious framework, Einstein and Langevin recognized that the behaviour of heavy particles in a gas can be studied by introducing two different scales: the inertial (slow) motion of the heavy particles and the interactions (fast) with the gas particles (Langevin, 1908). In geophysics the same approach has been - sometimes implicitly - applied for understanding important mechanisms driving the atmospheric and oceanic circulation: one can model the baroclinic instability because cyclones have a well-determined size and their structure emerges from the atmospheric turbulence (Charney, 1947), El Niño, because of the precise timescales involved in the phenomenon (Cane and Zebiak, 1985; Penland and Magorian, 1993). The success of a scale-separation-based approach is due to the intrinsic properties of stratified and rotating flows. In homogeneous and isotropic turbulence, the energy flows towards the small scales and coherent structures are rapidly destroyed. This is the so-called direct cascade proposed in (Kolmogorov, 1941). By contrast, geophysical flows are stratified and rotational flows, where an inverse cascade of energy induces the bidimensionalization of motions and contributes to the formation of large-scale coherent structures (Pouquet and Marino, 2013). In laboratory experiments (Lamriben et al., 2011) and geophysical observations (Craig and Banner, 1994; Holmes et al., 1998) one aims at separating coherent and turbulent components and build theoretical models to describe the associated motions (Charney, 1971; Pitcher, 1977; Jiang et al., 1995; Lucarini et al., 2007). As pointed out by several authors (see Schertzer and Lovejoy (1991) for a review), this task is non-trivial because both the inverse and direct cascades coexist for geophysical motions. The direct cascade is ultimately responsible for the dissipation of energy, the transfer of momentum from the atmosphere to the ocean and the soil, and the disruption of large- 
scale structures in the flow resulting in an unpredictable behaviour (Leith, 1971). The inverse cascade contributes to the formation of cyclonic and anticyclonic structures observed in the atmosphere and the ocean. Moreover, it generally enhances the predictability of future states of the atmosphere (Paladin and Vulpiani, 1994; Tribbia and Baumhefner, 2004).

In this paper we present two indicators that describe the statistical properties of large-scale coherent structures as well as turbulent spectra, investigating their response to climate change. The indicators are defined after separating the coherent structures from featureless turbulence via the wavelet filtering technique. For the coherent part, we compute the integral of the autocorrelation function as a measure of the persistence of the coherent structures. For the turbulent component, we use an indicator that measures the complexity of the spectrum with respect to the canonical behaviour theorized by Kolmogorov.

We test the technique on the horizontal wind data measured at $700 \mathrm{hPa}$ for two different anthropogenic emission scenarios (RCP 2.6 and 8.5). We investigate whether the anthropogenic and natural forcing could cause not only a change in the intensity of some defined observable (as it is now evident for the global mean temperature) but also in the direction the energy is cascading and therefore in the relative importance of large-scale coherent structure with respect to turbulence.

\section{Methods}

The separation between coherent $X(t)$ and turbulent $Y(t)$ components of a time series $Z(t)$ is done via wavelet filters (Farge, 1992). With respect to a simple filtering technique (e.g. moving-average filters), the wavelet filters are useful when the time series contain multiple timescales and there is no trivial scale separation. Wavelet analysis has been widely applied in climate science (Torrence and Compo, 1998) and to the analysis of geophysical time series (Ghil et al., 2002; Grinsted et al., 2004).

We give a brief illustration of the wavelet filtering technique by analysing a time series of $u_{500}$ taken from the scenario RCP 2.6 at the point $78^{\circ} \mathrm{W}, 38^{\circ} \mathrm{N}$. The series consists of 512 monthly observations and it is shown in Fig. 1a. Its power spectral density (psd) is visualized in Fig. 1b. The series shows an evident periodic component (the seasonal cycle) captured by the spectral peak. The spectrum is compared with a flat one (dotted line), reproducing a perfect whitenoise signal. The results of the wavelet filter are shown in Fig. 1c-f. The coherent component $X(t)$ and its spectrum are shown in Fig. 1c and d respectively. The effects of the filter are not directly visible on the detrended time series but rather on the spectra. The psd for the coherent part of the signal presents a significant slope with the energy concentrated on large timescales. By contrast, the incoherent component $Y(t)$ represented in Fig. 1e has a rather flat psd (Fig. 1f),
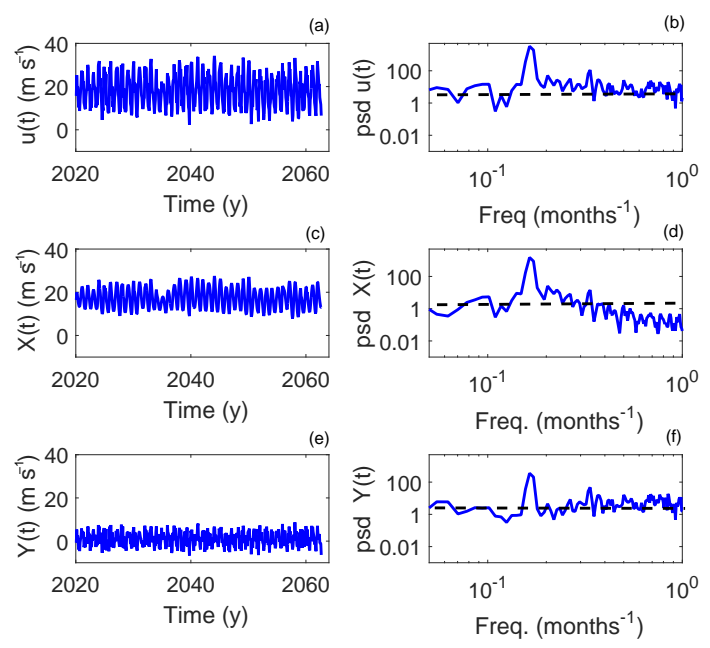

Figure 1. Example of wavelet filtering. (a) $u_{500}$ monthly time series at $78^{\circ} \mathrm{W}, 38^{\circ} \mathrm{N}$ and (b) corresponding power spectral density (psd). (c) Time series of the coherent component $X(t)$ extracted by the wavelet filter and (d) its psd. (e) Time series of the noisy component $Y(t)$ extracted by the wavelet filter and (f) its psd.

as expected from a successful application of the technique. Nonetheless, the limits of the wavelet filtering approach appear when one looks at the spectral peak corresponding to the seasonal cycle, which cannot be completely eliminated, although it represents a coherent component of the signal.

Once the separation between coherent and noisy component is done, we study the property of $X(t)$ and $Y(t)$ separately. For the coherent component $X(t)$ we use the memory of the system as an indicator of persistence by measuring the integral of the autocorrelation function (ACF) defined as

$\operatorname{ACF}(X)(\tau)=E\left[X(t) X^{*}(\tau)\right]$,

where $E[X]$ stands for expectation value. The ACF measures how long the system remember an initial condition. For a white-noise signal, it decays to 0 as $\tau>1$. For a correlated signal it decays slowly to 0 for large $\tau$. For a perfectly periodic signal, the ACF is periodic itself. The integral of the $\mathrm{ACF}$ in its discrete version is written as

$\Lambda=\sum_{\tau=0}^{T} \operatorname{ACF}(X)(\tau)$,

where we sum the correlation up to a time $T$ sufficiently large for the ACF to decay to $0 . \Lambda$ measures how long coherent structures persist in time, and it is therefore linked to predictability: the higher the correlation, the higher the probability that the structure will be preserved in future times (Schubert et al., 1992). The link between correlations decay and predictability is well known in dynamical systems theory and in physics (Osborne and Provenzale, 1989; Govindan et al., 1998; Crisanti et al., 1993), and we now exploit it to study properties of geophysical time series. 
For the noisy component $Y(t)$, we use an indicator of the spectral complexity with respect to the canonical Kolmogorov behaviour. In order to introduce this indicator we will use the class of autoregressive moving-average (ARMA) stochastic processes. In general, a stationary time series $Y(t)$ of an observable with unknown underlying dynamics can be modelled by an $\operatorname{ARMA}(p, q)$ process such that for all $t$

$Y_{t}=\sum_{i=1}^{p} \phi_{i} Y(t-i)+\varepsilon(t)+\sum_{j=1}^{q} \theta_{j} \varepsilon(t-j)$,

with $\varepsilon(t) \sim \mathrm{WN}\left(0, \sigma^{2}\right)-$ where $\mathrm{WN}$ stands for white noise - and the polynomials $\phi(z)=1-\phi_{1} z(t-1)-\ldots-\phi_{p} z(t-$ $p)$ and $\theta(z)=1-\theta_{1} z(t-1)-\ldots-\theta_{q} z(t-q)$. Notice that, hereinafter, the noise term $\varepsilon(t)$ will be assumed to be white noise, which is a very general condition (Box and Jenkins, 1970).

The basic model for the noisy component is the $\operatorname{ARMA}(1,0)$ or simply AR(1) model, which is the simplest model compatible with the Kolmogorov spectrum (Thomson, 1987). When the spectral complexity increases, the best ARMA model describing the velocity time series will deviate from the basic one. We can define a normalized distance between the reference $\operatorname{ARMA}(\bar{p}, \bar{q})$ and any other $\operatorname{ARMA}(p$, $q$ ) by using the Bayesian information criterion (BIC), which measures the relative quality of a statistical model, as

$\mathrm{BIC}=-2 \ln \hat{L}\left(n, \hat{\sigma}^{2}, p, q\right)+k[\ln (n)+\ln (2 \pi)]$,

where $\hat{L}\left(n, \hat{\sigma}^{2}, p, q\right)$ is the likelihood function for the investigated model and in our case $k=p+q$ and $n$ is the length of the sample. The variance $\hat{\sigma}^{2}$ is computed from the sample and is a series-specific quantity.

Our indicator is a normalized difference between the $\operatorname{BIC}\left(n, \hat{\sigma}^{2}, p+1, q\right)$ and the $\operatorname{ARMA}(\bar{p}, \bar{q}) \operatorname{BIC}\left(n, \hat{\sigma}^{2}, \bar{p}, \bar{q}\right):$

$\Upsilon=1-\exp \{|\operatorname{BIC}(p+1, q)-\operatorname{BIC}(\bar{p}, \bar{q})|\} / n$,

with $0 \leq \Upsilon \leq 1$; it goes to 0 if the dataset is well described by an $\operatorname{ARMA}(\bar{p}, \bar{q})$ model and tends to 1 in the opposite case.

We have checked that such indicators perform well in different physical systems and generally provide more information than the ones based on correlations analysis only. Such results can been found in Faranda et al. (2014), where indicators similar to $\Upsilon$ have been used to model different physical systems: Ising and Langevin models and turbulence. A large $\Upsilon$ corresponds to a complex spectrum with non-trivial scale interactions and non-constant energy transfers; a small $\Upsilon$ corresponds to a spectrum compatible with the Kolmogorov spectrum with constant energy fluxes between scales. The predictability will decrease with a higher $\Upsilon$ because more structures on different scales will have to be taken into account to describe the behaviour of the system. In other words, for high $\Upsilon$ the component $Y(t)$ cannot just be modelled as simple noise.

\section{Analysis}

We illustrate the potential of $\Lambda$ and $\Upsilon$ indicators on a climate change experiment used in the CMIP5 framework for the IPCC AR5 report (Collins et al., 2013). To explore climate change in the next century, the IPCC has developed four different scenarios, defined in terms of radiative evolution and corresponding to a concentration of greenhouse gases year by year between 2006 and 2100, extended until 2300. Here we consider two scenarios: (i) the low-emission scenario (RCP 2.6), leading to a radiative balance of $2.6 \mathrm{~W} \mathrm{~m}^{-2}$ in 2100 with a peak at $3 \mathrm{~W} \mathrm{~m}^{-2}$ and a decreasing trend; (ii) the higher-emission scenario (RCP 8.5), predicting an increase up to $8.5 \mathrm{~W} \mathrm{~m}^{-2}$ in 2100 . The effect of such greenhouse gas perturbations are well known for some observables, e.g. the global temperature increase ranges from $1 \pm 0.4$ to $3.7 \pm 0.7^{\circ} \mathrm{C}$ in the last part of the period 2081-2100 (Collins et al., 2013).

We focus on the daily horizontal winds at $700 \mathrm{hPa}$ $\left(u_{700}, v_{700}\right)$ obtained from the IPSLCM5-LR model, where LR stands for low resolution. This model is developed by the Institut Pierre-Simon Laplace with several laboratories. It consists of several components: atmosphere (LMDZ), ocean (NEMO), continent (ORCHIDEE), and sea ice (LIM). For LMDZ and ORCHIDEE, the spatial resolution is $3.75^{\circ} \times 1.875^{\circ}$ (longitude $\times$ latitude) with 39 vertical levels. For NEMO, the resolution is of about $2^{\circ}$, with a higher latitudinal resolution of $0.5^{\circ}$ in the equatorial ocean and 31 vertical levels. ORCHIDEE takes into account the evolution of the lands (urbanization, forests, agriculture) (Dufresne et al., 2013). We chose $700 \mathrm{hPa}$ (about $3 \mathrm{~km}$ ) because it has been recognized as the best level for tracking the coherent atmospheric structures as shortwaves, extratropical cyclones, and convective storms, since they are all advected, in first approximation, by $700 \mathrm{hPa}$ horizontal winds (Mölders and Kramm, 2014). We have also performed robustness tests by considering the medium-resolution (MR) version of the IPSLCM5 model $\left(2.5^{\circ} \times 1.25^{\circ}\right.$ in longitude and latitude for LMDZ and ORCHIDEE, while the resolution of other models is the same as LR). These tests are reported and explained in the Supplement.

We begin the analysis by showing typical maps of $\Lambda$ and $\Upsilon$ for the scenario RCP 2.6 and the two components: the zonal $u_{700}$ and the meridional $v_{700}$ (Fig. 2). $\Lambda$ shows, for the zonal component, a rich structure with several areas where persistent coherent structures are well identified (Fig. 2a). Because we are using daily time series, the values of $\Lambda$ can be directly interpreted as the number of days of persistence of these structures. At the midlatitudes, the signature of stationary planetary waves is visible. In correspondence to the location of such waves, we find $\Lambda \sim 16$ days, a value compatible with the work of Torrence and Compo (1998), who studied planetary waves using other indicators based on the wavelet approach. In the tropics, the high values of $\Lambda$ can be linked to the easterly jets (Koteswaram, 1958). The core of 
(a)

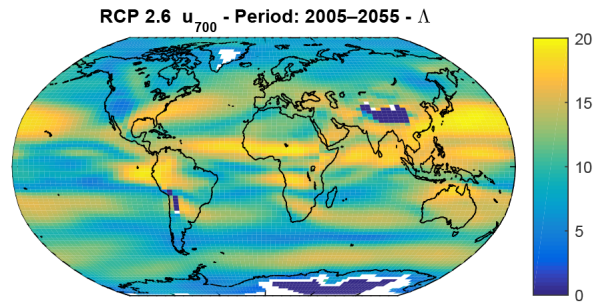

(c)

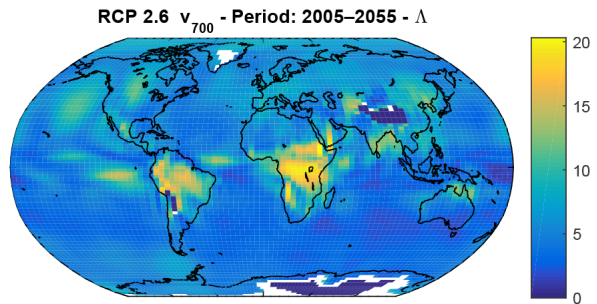

(b)

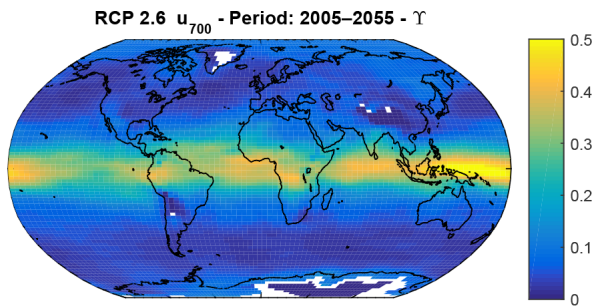

(d)

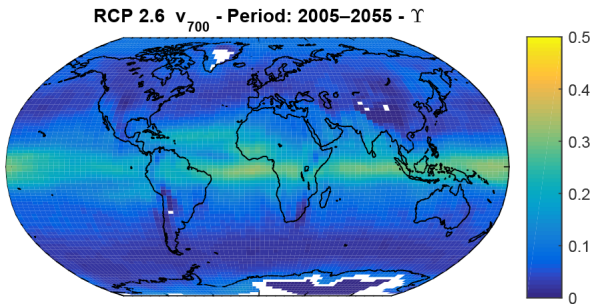

Figure 2. Integral of the autocorrelation function $\Lambda$ for the period 2005-2055 for the $u_{700}$ (a) and for the $v_{700}$ (c) daily time series. Spectral complexity $\Upsilon$ for the period 2005-2055 for the $u_{700}$ (b) and for the $v_{700}$ (d) daily time series.

the African easterly jet is located at about this level (Nicholson, 2009), as are the Choco and Caribbean low-level jets (Wang, 2007). The results for $\Lambda$ computed on the meridional component $v_{700}$ are shown in Fig. 2c. Here the largest values are found in correspondence to the regions affected by monsoons. The strongest signal is for the African monsoon because the IPSL model localizes it better than the Indian one (Dufresne et al., 2013). At the midlatitudes the patches visible near the Pacific coast and over the USA correspond to areas where the zonal flow is blocked by the Rocky Mountains and meridional winds blow to allow the flows go round the mountains.

For the $\Upsilon$ analysis, there is not much difference in the structure of the zonal component (Fig. 2b) and the meridional component (Fig. 2d). The spatial pattern of $\Upsilon$ can be explained in light of the tropical atmospheric dynamics. Higher values are located in the tropics, where turbulence is associated with the convective activity in the area. We note that, as the resolution is increased, the $\Delta \Upsilon$ increases (see Fig. 7 in the Supplement). This means that turbulent contribution becomes more relevant when finer scales are included in the analysis, although the scale of the analysis remains, even in the MR simulation, much larger than the scales of convective structures $(\sim 10 \mathrm{~km})$.

We now investigate whether $\Lambda$ and $\Upsilon$ can detect changes in the coherent or noisy components of the $700 \mathrm{hPa}$ horizontal winds under the climate change RCP 2.6 and RCP 8.5 scenarios. We divide the daily time series into two periods (2005-2055 and 2055-2105) and compute the quantities $\Delta \Lambda=\Lambda_{2055-2105}-\Lambda_{2005-2055}$ and $\Delta \Upsilon=\Upsilon_{2055-2105-}$ $\Upsilon_{2005-2055}$ (Fig. 3). For both the indicators, the RCP 8.5 scenario shows considerable impacts, whereas the changes for the RCP 2.6 are appreciable only for the $u_{700}$ component. In the Supplement, we report the results also for time windows of 30 and 40 years. By decreasing the time window considered, we observe that the spatial structure of $\Delta \Lambda$ and $\Delta \Upsilon$ does not change (Figs. 1-6 in the Supplement). However, the changes in the indicator become locally sharper as shorter time windows are considered, as one would expect by taking differences of periods with a larger time separation (Fig. 7 in the Supplement).

The $\Delta \Lambda$ fields for the $u_{700}$ and the $v_{700}$ components present interesting structures: the strongest signals are located over the Pacific Ocean in correspondence to the El Niño Region 3 (for $u_{700}$ ) and Region 3.4 for $v_{700}$, described in Trenberth (1997). At the midlatitudes, dipolar structures appear both for the zonal and for the meridional flow (Barnston et al., 1997). This is the signature of the jet stream shift observed by several studies. For the Northern Hemisphere, changes in the pattern of meridional winds are highlighted by an alternation of negative and positive $\Delta \Lambda$ : we can link these changes to the modification in stationary planetary waves associated with the change in the jet stream intensity and positions. Several studies have recently appeared on this issue, although is not clear whether the cause can be linked to the so-called Arctic amplification (Serreze and Francis, 2006) rather than to changes in El Niño-Southern Oscillation (Moritz et al., 2002) or even to the stratospheric dynamics (Serreze and Barry, 2011). $\Delta \Lambda$ also suggests that some regions will experience an increase in persistence of meridional winds, i.e. an increasing of blocking conditions (e.g. central USA, western Europe), whereas some other areas will have an increase in the persistence of the zonal winds (e.g. eastern Europe, Alberta (CA)).

The results for $\Delta \Upsilon$ indicate that the spectral complexity in the tropical regions tends to reduce by about $10 \%$ $(\Delta \Upsilon \sim-0.1)$ in the RCP 8.5 scenario. Although the model does not resolve the convective scales, a possible explana- 
(a)

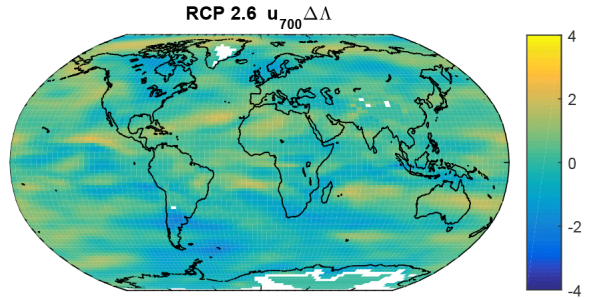

(c)

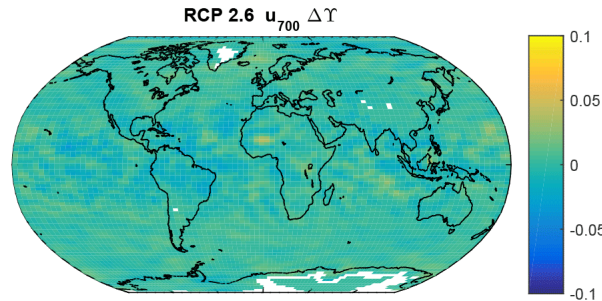

(e)

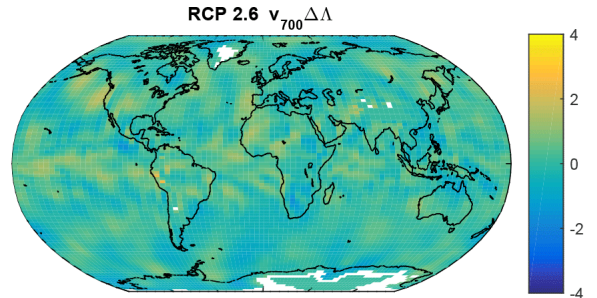

(g)

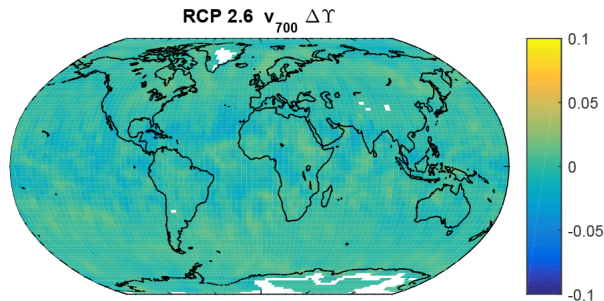

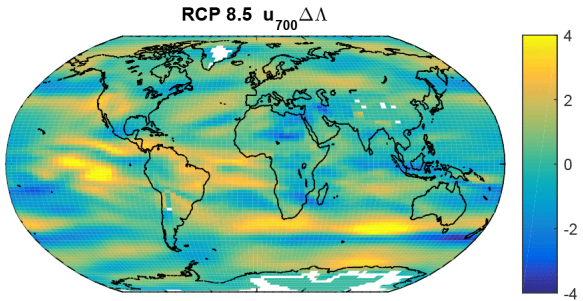

(d)
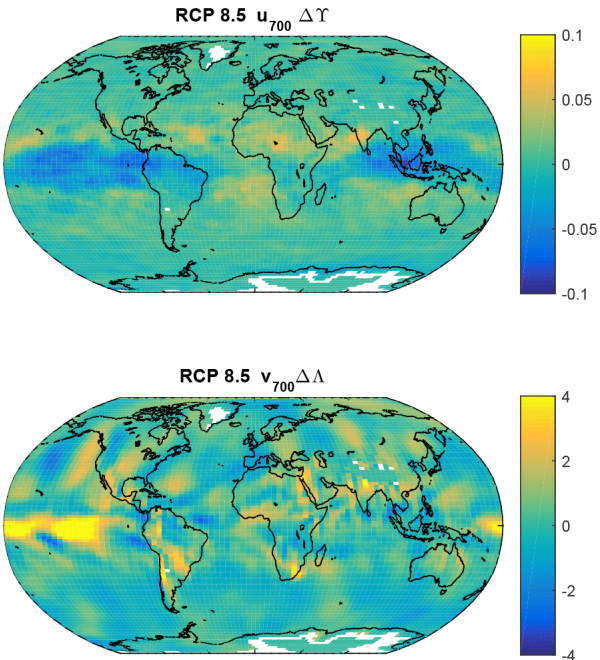

(h)

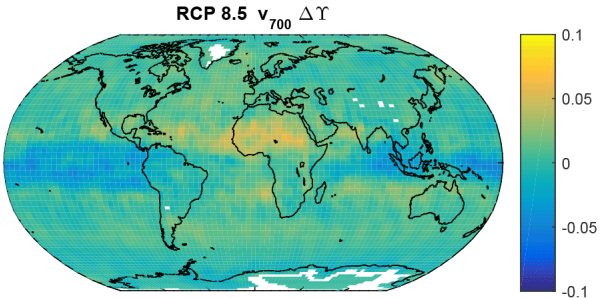

Figure 3. Differences in the integral of the autocorrelation function $\Delta \Lambda$ for the 2055-2105 and the 2005-2055 period for the $u_{700}$ in the RCP 2.6 (a) and in the RCP 8.5 (b). Differences in the spectral complexity $\Delta \Upsilon$ for the 2055-2105 and the 2005-2055 period for the $u_{700}$ in the RCP 2.6 (c) and RCP 8.5 (d) scenario. $\Delta \Lambda$ for $v_{700}$ for the RCP 2.6 (e) and the RCP 8.5 (f) scenarios. $\Delta \Upsilon$ for the $v_{700}$ in the RCP 2.6 (g) and RCP 8.5 (h) scenario.

tion for this result relies on the enhanced convective activity resulting from the sea surface warming and resulting in an increase in precipitation in the area, as we have verified for the IPSL model and as reported by other studies (Huang et al., 2013). This consideration is valid only if convective parametrization transfers energy from the convective scales to the scale of the analysis. In other words, at the actual resolution of climate models, we can only observe the footprints of these phenomena. If turbulence becomes stronger and anisotropic, then it should approach the Kolmogorov behaviour and $\Upsilon$ should tend to 0 .

\section{Conclusions}

We have devised two indicators to study the changes in the atmospheric circulation by separating the coherent structures from the turbulent part of the signals analysed, i.e the velocity fields at $700 \mathrm{hPa}$. The indicator $\Delta \Lambda$ is a measure of the total persistence of the coherent structures, whereas $\Delta \Upsilon$ is a measure of the residual complexity of the turbulent spectrum, once the coherent component has been removed.

The indicators show significant changes when the climate system is subject to greenhouse gas forcing. The difference in the indicators for the RCP 8.5 scenario between the second half and the first half of the 21st century suggests that El Niño-Southern Oscillation will play a major role and block- 
ing conditions will change the trajectory of coherent structures observed at the midlatitudes.

Besides the regional patterns, we believe that the most important message is contained in the global average of our indicators. For the RCP 8.5 scenario, $\Delta \Lambda$ increases by 0.5 days in the second half of the century for $u_{700}$ and by 0.2 days for $v_{700}$. On the other hand, the spectral complexity decreases by about $10 \%$ in the tropical regions. This suggests that the coherent structures will play a major role in the atmospheric dynamics. This will probably result in an enhanced predictability of the atmosphere on weekly to monthly timescales. The contrast between these two effects could be one of the causes of the difficulty in finding significant traces of climate change in the circulation dynamics, a problem recently highlighted by Shepherd (2014).

Our indicators have been here illustrated for a single model at two different spatial resolutions and in two climate change scenarios. They could be useful to evaluate the response of atmospheric circulation to changes in the forcing for several models. Moreover, the technique does not require the variables to be velocity fields and it could be extended to any physical time series in which a non-trivial scale separation is present.

\section{The Supplement related to this article is available online at doi:10.5194/esd-7-517-2016-supplement.}

Acknowledgements. Davide Faranda acknowledges Berengere Dubrulle, Pascal Yiou, and Mathieu Vrac for useful comments and discussion. Davide Faranda was supported by ERC grant No. 338965-A2C2. Dimitri Defrance was supported by the French Atomic Commission (CEA) within the framework of the VACCIN project (Variations Abruptes du Climat: Conséquences et Impacts éNergétiques) funded by the DSM-Energie Program. It benefited from the HPC resources made available by GENCI (Grand Equipement National de Calcul Intensif), CEA, and CNRS (Centre National de la Recherche Scientifique).

Edited by: B. Kravitz

\section{References}

Barnston, A. G., Chelliah, M., and Goldenberg, S. B.: Documentation of a highly ENSO-related SST region in the equatorial Pacific, Atmos.-Ocean., 35, 367-383, 1997.

Box, G. E. and Jenkins, G. M.: Time Series Analysis: Forecasting and Control, Holden-D. iv, 1970.

Cane, M. A. and Zebiak, S. E.: A theory for El Nino and the Southern Oscillation, Science, 228, 1085-1087, 1985.

Charney, J. G.: The dynamics of long waves in a baroclinic westerly current, J. Meteorol., 4, 136-162, 1947.

Charney, J. G.: Geostrophic turbulence, J. Atmos. Sci., 28, 10871095, 1971.
Collins, M., Knutti, R., Arblaster, J., Dufresne, J., Fichefet, T., Friedlingstein, P., Gao, X., Gutowski, W., Johns, T., Krinner, G., Shongwe, M., Tebaldi, C., Weaver, A. J., and Wehner, M.: Climate change 2013: the physical science basis. Contribution of Working Group I to the Fifth Assessment Report of the Intergovernmental Panel on Climate Change, Long-term Climate Change: Projections, Commitments and Irreversibility, Cambridge Univ. Press, Cambridge, UK, and New York, 1029-1136, 2013.

Craig, P. D. and Banner, M. L.: Modeling wave-enhanced turbulence in the ocean surface layer, J. Phys. Oceanogr., 24, 25462559, 1994.

Crisanti, A., Jensen, M., Vulpiani, A., and Paladin, G.: Intermittency and predictability in turbulence, Phys. Rev. Lett., 70, 166-169, 1993.

Dufresne, J.-L., Foujols, M.-A., Denvil, S., Caubel, A., Marti, O., Aumont, O., Balkanski, Y., Bekki, S., Bellenger, H., Benshila, R., et al.: Climate change projections using the IPSL-CM5 Earth System Model: from CMIP3 to CMIP5, Climate Dynamics, 40, 2123-2165, 2013.

Faranda, D., Pons, F. M. E., et al: Statistical early-warning indicators based on autoregressive moving-average models, J. Phys. A, 47, 252001, 2014.

Farge, M.: Wavelet transforms and their applications to turbulence, Ann. Rev. Fluid Mech., 24, 395-458, 1992.

Ghil, M., Allen, M., Dettinger, M., Ide, K., Kondrashov, D., Mann, M., Robertson, A. W., Saunders, A., Tian, Y., Varadi, F., and P. Yiou : Advanced spectral methods for climatic time series, Rev. Geophys., 40, 1003, doi:10.1029/2000RG000092, 2002.

Govindan, R., Narayanan, K., and Gopinathan, M.: On the evidence of deterministic chaos in ECG: Surrogate and predictability analysis, Chaos: An Interdisciplinary Journal of Nonlinear Science, 8, 495-502, 1998.

Grinsted, A., Moore, J. C., and Jevrejeva, S.: Application of the cross wavelet transform and wavelet coherence to geophysical time series, Nonlin. Processes Geophys., 11, 561-566, doi:10.5194/npg-11-561-2004, 2004.

Holmes, P., Lumley, J. L., and Berkooz, G.: Turbulence, coherent structures, dynamical systems and symmetry, Cambridge University Press, 1-420, 1998.

Huang, P., Xie, S.-P., Hu, K., Huang, G., and Huang, R.: Patterns of the seasonal response of tropical rainfall to global warming, Nature Geosci., 6, 357-361, 2013.

Jiang, S., Jin, F.-F., and Ghil, M.: Multiple equilibria, periodic, and aperiodic solutions in a wind-driven, double-gyre, shallow-water model, J. Phys. Oceanogr., 25, 764-786, 1995.

Kolmogorov, A. N.: Dissipation of energy in locally isotropic turbulence, : Dokl. Akad. Nauk SSSR, 32, 16-18, 1941.

Koteswaram, P.: The easterly jet stream in the tropics, Tellus, 10, 43-57, 1958.

Lamriben, C., Cortet, P.-P., and Moisy, F.: Direct measurements of anisotropic energy transfers in a rotating turbulence experiment, Phys. Rev. Lett., 107, 024503, doi:10.1103/PhysRevLett.107.024503, 2011.

Langevin, P.: Sur la théorie du mouvement brownien, C. R. Acad. Sci. Paris, 146, 530-533, 1908.

Leith, C.: Atmospheric predictability and two-dimensional turbulence, J. Atmos. Sci., 28, 145-161, 1971. 
Lucarini, V., Speranza, A., and Vitolo, R.: Self-scaling of the statistical properties of a minimal model of the atmospheric circulation, in: Nonlinear Dynamics in Geosciences, 197-219, Springer, 2007.

Mölders, N. and Kramm, G.: Lectures in Meteorology, Springer, 1-591, 2014.

Moritz, R. E., Bitz, C. M., and Steig, E. J.: Dynamics of recent climate change in the Arctic, Science, 297, 1497-1502, 2002.

Murray, C. D. and Dermott, S. F.: Solar system dynamics, Cambridge University Press, 1-608, 1999.

Nicholson, S. E.: A revised picture of the structure of the "monsoon" and land ITCZ over West Africa, Climate Dynamics, 32, 1155-1171, 2009.

Osborne, A. R. and Provenzale, A.: Finite correlation dimension for stochastic systems with power-law spectra, Physica D, 35, 357381, 1989

Paladin, G. and Vulpiani, A.: Predictability in spatially extended systems, J. Phys. A, 27, 4911, doi:10.1088/03054470/27/14/018, 1994.

Penland, C. and Magorian, T.: Prediction of Nino 3 sea surface temperatures using linear inverse modeling, J. Climate, 6, 10671076, 1993.

Pitcher, E. J.: Application of stochastic dynamic prediction to real data, J. Atmos. Sci., 34, 3-21, 1977.

Pouquet, A. and Marino, R.: Geophysical turbulence and the duality of the energy flow across scales, Phys. Rev. Lett., 111, 234501, doi:10.1103/PhysRevLett.111.234501, 2013.
Schertzer, D. and Lovejoy, S.: Nonlinear variability in geophysics, Springer, 1-318, doi:10.1007/978-94-009-2147-4, 1991.

Schubert, S. D., Suarez, M. J., and Schemm, J.-K.: Persistence and predictability in a perfect model, J. Atmos. Sci., 49, 256-269, 1992.

Serreze, M. C. and Barry, R. G.: Processes and impacts of Arctic amplification: A research synthesis, Global Planet. Change, 77, 85-96, 2011.

Serreze, M. C. and Francis, J. A.: The Arctic amplification debate, Clim. Change, 76, 241-264, 2006.

Shepherd, T. G.: Atmospheric circulation as a source of uncertainty in climate change projections, Nature Geosci., 2014.

Thomson, D.: Criteria for the selection of stochastic models of particle trajectories in turbulent flows, J. Fluid Mech., 180, 529-556, 1987.

Torrence, C. and Compo, G. P.: A practical guide to wavelet analysis, B. Am. Meteor. Soc., 79, 61-78, 1998.

Trenberth, K. E.: The definition of el nino, B. Am. Meteor. Soc., 78, 2771-2777, 1997.

Tribbia, J. and Baumhefner, D.: Scale interactions and atmospheric predictability: An updated perspective, Mon. Weather Rev., 132, 703-713, 2004.

Wang, C.: Variability of the Caribbean low-level jet and its relations to climate, Clim. Dynam., 29, 411-422, 2007. 